\title{
SQUARE STEPPING EXERCISE E EXERCÍCIOS BÁSICOS NA COGNIÇÃO DE IDOSOS
}

\author{
Camila Vieira Ligo Teixeira* \\ Sebastião Gobbi** \\ Jessica Rodrigues Pereira*** \\ DeisyTerumiUeno*** \\ RyosukeShigematsu**** \\ Lilian Teresa BuckenGobbi*****
}

\begin{abstract}
RESUMO
O objetivo do presente estudo foi comparar as funções cognitivas de idosos antes e após dois protocolos de intervenção: exercícios básicos combinados com o Square SteppingExercise (COM) e apenas exercícios básicos (EB). Todos foram avaliados antes e depois dos 4 meses de intervenção por meio do Montreal CognitiveAssessment (MoCA) e do Teste Winsconsin de Classificação de Cartas (TWCC). Os resultados mostraram que ambos os grupos melhoraram o estado cognitivo global, mas que apenas o COM apresentou melhora específica em abstração e flexibilidade mental. Assim, o treinamento combinado de exercícios básicos e SSE parece ser mais efetivo para a cognição quando comparado com um treinamento exclusivamente com exercícios básicos.
\end{abstract}

Palavras-Chave: Idosos. Cognição. Exercício físico.

\section{INTRODUÇÃO}

Pesquisas recentes sobre o envelhecimento têm mostrado os benefícios da prática do exercício físico nas funções cognitivas (CHRISTOFOLETTI et al., 2008; ETNIER et al., 2006; ANTUNES et al., 2001; CASSILHAS et al., 2007; COLCOMBE; KRAMER, 2003). Em consequência, tais estudos têm fundamentado a hipótese de que a prática do exercício físico pode contribuir para a prevenção de declínio cognitivo (CASSILHAS et al., 2007; ANTUNES et al., 2006; LAURIN et al., 2001). Estas pesquisas revelam possibilidades para manutenção da vitalidade cognitiva durante o envelhecimento, por meio do engajamento social, estimulação intelectual e prática regular de atividades físicas (BUTLER et al., 2004; VANCE et al., 2005; VERGHESE et al., 2003).

Além da preservação das funções cognitivas no envelhecimento, manter um nível adequado da capacidade funcional pode garantir autonomia e independência aos idosos (GUIMARÃESet al., 2004). A prática regular da

\footnotetext{
* Mestre em Ciências da Motricidade. Instituto de Biociências, Universidade Estadual Paulista, Departamento de Educação Física, Laboratório de Atividade Física e Envelhecimento (LAFE).

** Professor Doutor. Instituto de Biociências, Universidade Estadual Paulista, Departamento de Educação Física, Laboratório de Atividade Física e Envelhecimento (LAFE).

*** Mestranda em Ciências da Motricidade. Instituto de Biociências, Universidade Estadual Paulista, Departamento de Educação Física, Laboratório de Atividade Física e Envelhecimento (LAFE).

**** Mestranda em Ciências da Motricidade. Instituto de Biociências, Universidade Estadual Paulista, Departamento de Educação Física, Laboratório de Atividade Física e Envelhecimento (LAFE). ****** Professor Doutor. Faculdade de Educação, MieUniversity.

****** Professora Doutora. Instituto de Biociências, Universidade Estadual Paulista, Departamento de Educação Física, Laboratório de Estudos da Postura (LEPLO).
} 
atividade física é uma das principais formas de minimizar os declínios físicos, psicológicos e sociais que podem estar associados ao envelhecimento (GOBBI, 1997; BENEDETTI et al., 2008).

Há evidências de que diversos tipos de treinamento físico melhoram os componentes da capacidade funcional em idosos (SEBASTIÃO et al., 2008; GONÇALVES et al., 2007; MAZO et al., 2006). Recentemente, nosso grupo de pesquisa introduziu no Brasil uma nova modalidade de programa de treinamento - o Square SteppingExercise(SSE). O SSE foi criado por Shigematsu e Okura (2006), com o intuito de melhorar o equilíbrio de seus praticantes, diminuindo assim o risco de quedas. É um programa baseado em passos sobre um tapete de 2,5 x 1,0 m, com várias sequências mostradas pelo profissional, as quais devem ser visualizadas e posteriormente repetidas pelos participantes, sem consulta a qualquer tipo de desenho ou escrita. A cada sequência, a dificuldade aumenta. Por exemplo, os passos que iam apenas para frente, vão para o lado, para trás, na diagonal. O SSE tem sido eficaz na melhoria dos componentes da capacidade funcional, como equilíbrio, força de membros inferiores, flexibilidade e agilidade, diminuindo, consequentemente, 0 risco de quedas (SHIGEMATSU; OKURA, 2006; SHIGEMATSU et al., 2008a). Além disso, o SSE parece demandar um alto nível de funções cognitivas, fundamentando a hipótese de que sua prática regular possa também beneficiar tais funções, o que seria particularmente importante em pessoas idosas.

Desta forma, o objetivo do presente estudo foi analisar e comparar possíveis efeitos, nas funções cognitivas de idosos, de dois protocolos de intervenção: exercícios básicos associados ao SSE e apenas exercícios básicos.

\section{METODOLOGIA}

\section{Amostra e recrutamento}

Foram selecionadas para este estudo 56 pessoas idosas (60 anos ou mais) de ambos os gêneros, não institucionalizadas e com deambulação independente. O recrutamento foi feito por meio da divulgação (jornal, cartaz, visita a grupos de terceira idade, contato telefônico) e os idosos foram distribuídos de forma aleatória em dois grupos de treinamento: Exercícios 
Básicos (EB) e Combinado (COM; Square SteppingExercise(SSE) combinado com EB). Aqueles que aceitaram participar assinaram um Termo de Consentimento Livre e Esclarecido, como parte do projeto de pesquisa aprovado pelo Comitê de Ética em Pesquisa do Instituto de Biociências, UNESP, campus Rio Claro (Protocolo $\mathrm{n}^{-}$4798/2009).

$O$ estudo foi concluído com $n=25$ no $\operatorname{COM}$ e $n=20$ no EB. Foram excluídos do estudo pessoas idosas que apresentavam pontuação no Mini Exame do Estado Mental abaixo da nota de corte, de acordo com os critérios de Bruckiet al. (2003), e aqueles que tiveram assiduidade às sessões de treinamento menor que $75 \%$.

\section{Protocolo de Avaliação}

Para caracterização da amostra foi utilizado o Questionário de Baecke Modificado para Idosos(QBMI), proposto e validado por Voorripset al. (1991), que abrange três dimensões básicas: atividades domésticas, atividades esportivas e atividades de lazer, a fim de estimar o nível de atividade física dos participantes.

Foi utilizado o Montréal CognitiveAssessment (MoCA - SMITH et al., 2007), para avaliar o estado cognitivo global dos participantes, acessando diferentes domínios cognitivos. Esse teste foi aplicado antes e depois do período experimental.

Com o intuito de avaliar as funções executivas, foi utilizado o Teste Wisconsin de Classificação de Cartas - Modificado (TWCC)(NELSON, 1976; adaptado de BERG, 1948). O teste é composto por quatro cartões estímulo e 48 cartões-resposta, os quais devem ser combinados aos cartões estímulo. Seguindo a dica do experimentador ("certo" ou "errado"), o avaliado deveria descobrir a regra de combinação (cor, forma ou número dos desenhos em cada cartão-estímulo). Foi avaliado o número de categorias completadas (equivalente a seis acertos consecutivos) e o número de erros perseverativos (quando este é recorrente, mesmo após o experimentador avisar que está errado). Este teste avalia as funções executivas (SPREEN; STRAUSS, 1991; LEZAK, 2004) e apresenta validade para tal (BURGESS et al., 1998). 


\section{Protocolo de Treinamento}

Ambos os grupos que receberam intervenção por um período de 4 meses, com sessões de 40 minutos cada, de intensidade moderada e frequência de três vezes por semana.

O treinamento do COM foi composto por exercícios básicos (embasado nas recomendações da ACSM, 2009) e pelas sequências do SSE (SHIGEMATSU; OKURA, 2006). As sessões foram de intensidade moderada e compostas, por ordem, de:

a) 5 minutos de aquecimento com alongamento muscular;

b) 15 minutos de exercícios (sem grande demanda de funções cognitivas, coordenação motora e ritmo) que estimulassem a capacidade aeróbia, flexibilidade, resistência muscular localizada e equilíbrio. Os exercícios eram padronizados e repetitivos, sem o envolvimento de atividades combinadas ou complexas, ou seja, exercícios que não exigissem grande atenção, memorização, resolução de problemas (caminhada normal; caminhada elevando o joelho, elevando os braços, mudando de direção, elevando os calcanhares, de costas; flexão de joelhos; elevação de calcanhares segurando na barra; flexão de quadril apoiando-se em barra; flexão de cotovelo; abdução de ombro). Foi iniciado com 3 séries de 10 repetições e, progressivamente, atingiu 3 séries de 30 repetições, no decorrer do treinamento;

c) Na metade final da sessão (20 minutos) foi realizado o SSE, utilizando-se um tapete para cada grupo de 8 participantes, sendo o desempenho dos participantes monitorado por 1 orientador por grupo. O orientador demonstrava 3 vezes cada sequência, antes dos participantes tentarem executá-la. As sequências foram compostas de passos para frente, para trás, para a lateral e para a diagonal e, a cada sequência, a complexidade da combinação dos passos aumentava, como ilustra, a título de exemplo, a Figura 1. Os participantes foram encorajados a se concentrarem na sequência, para que tivessem sucesso no desempenho da mesma e das outras mais complexas. Não foi utilizada qualquer outra dica visual (que não a demonstração pelo orientador) ou auditiva. 
Categorias das sequências

\begin{tabular}{|c|c|c|c|c|c|c|c|c|c|c|c|c|}
\hline \multicolumn{2}{|c|}{ Elementar } & \multicolumn{4}{|c|}{ Básico } & \multicolumn{3}{|c|}{ Intermediário 01} & \multicolumn{4}{|c|}{ Avançado 02} \\
\hline 2 & & 4 & 3 & (1) & (3) & 6 & 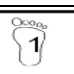 & 5 & 2 & 6 & 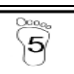 & 1 \\
\hline & [1] & 4 & 3 & [1] & (3) & 4 & 3 & 3 & 8 & 4 & 3 & [7] \\
\hline 2 & & 4 & 3 & (1) & 3 & 6 & 1 & 5 & \begin{tabular}{|l|}
599 \\
669
\end{tabular} & {$\left[\begin{array}{c}9 \\
2\end{array}\right.$} & {$\left[\begin{array}{l}09 \\
11\end{array}\right.$} & 5 \\
\hline & {$[1]$} & {$[4$} & 3 & (1) & (3) & {$[4$} & 3 & (3) & \begin{tabular}{|l|l|}
39 \\
of
\end{tabular} & 7 & 3 & 9 \\
\hline 2 & & 4 & 3 & 1 & 3 & 6 & 1 & 5 & 2 & 6 & 5 & 1 \\
\hline & {$[1$} & 4 & 2 & (1) & 3 & 4 & 3 & 3 & 8 & 4 & 3 & 7 \\
\hline 2 & & 4 & 2 & ] & 3 & 6 & 1 & 5 & $\mid 69$ & {$\left[\begin{array}{l}9 \\
2\end{array}\right.$} & {$\left[\begin{array}{l}1 \\
1\end{array}\right.$} & 5 \\
\hline & (1) & 4 & 3 & $1]$ & (3) & 44 & {$[3$} & (3) & \begin{tabular}{|l|}
10 \\
0
\end{tabular} & 4 & 3 & 9 \\
\hline 2 & & 4 & 3 & (1) & 3 & 6 & 1 & 5 & 2 & 6 & 5 & 1 \\
\hline & 1 & 4 & 2 & 1 & 3 & 4 & 2 & 3 & 8 & 4 & 3 & 7 \\
\hline
\end{tabular}

Figura 1. Esquema ilustrativo de sequências de andar sobre o tapete do Square SteppingExercise. (Adaptado de SHIGEMATU; OKURA, 2006)

O treinamento do EB foi similar ao do COM, exceto que não envolvia o SSE, a segunda parte das sessões (exercícios básicos) tinha duração de 30 minutos e foi incluída uma parte de volta à calma de 5 minutos, ao final.

\section{Processamento e Análise dos Dados}

Inicialmente os dados foram tratados a partir de procedimentos estatísticos descritivos (média e desvio-padrão) e, para ilustrar o comportamento das variáveis, foi calculado o delta percentual de cada variável. Após analisada a normalidade da distribuição pelo teste de Shapiro-Wilk, foi utilizado o Teste $t$ independente para verificar possíveis diferenças entre variáveis de caracterização da amostra e de controle. A Anova two-way, tendo como fatores "grupo" e "momento", foi empregada para comparação do comportamento dos grupos e possíveis efeitos da intervenção. O nível de significância adotado foi de $5 \%(p<0,05)$.

\section{RESULTADOS}

Não foram encontradas diferenças significativas $(p>0,05)$ entre is grupos nos dados de caracterização da amostra e nas variáveis dependentes, no momentos inicial (pré) da pesquisa, como mostra a Tabela 1 e 2. 
Tabela 1. Valores médios e desvios-padrão de caracterização (idade, escolaridade, nível de atividade física) dos participantes por grupo.

\begin{tabular}{|c|c|c|c|}
\hline Grupos & $\operatorname{COM}(n=25)$ & EB $(n=20)$ & \\
\hline & Média土DP & 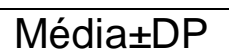 & $P$ \\
\hline Idade (anos) & $67,1 \pm 5,8$ & $66,9 \pm 5,3$ & 0,61 \\
\hline $\begin{array}{l}\text { Escolaridade } \\
\text { (anos) }\end{array}$ & $5,7 \pm 3,9$ & $6,2 \pm 4,1$ & 0,58 \\
\hline QBMI (pontos) & $3,1 \pm 1,8$ & $3,4 \pm 1,8$ & 0,99 \\
\hline
\end{tabular}

Houve interação significativa na variável dependente errosperseverativos do TWCC $(F=15,03 ; \quad p<0,001)$, mostrando que o SSE contribuiu significativamente para tal melhora, enquanto o EB cometeu mais erros após a intervenção. Foi observado melhora significativa entre os momentos pré e pósintervenção, para ambos os grupos, no MoCA e, apenas para o COM, na variável número de categorias do TWCC, como mostra a Tabela 2. Conquanto não tenha sido observada interação significativa nas variáveis, os valores de delta percentual mostram que o SSE apresentou maiores mudanças nas variáveis do estudo do que o EB.

Tabela 2. Resultados médios e desvios-padrão no Montreal CognitiveAssessment (MoCA) e Teste Wisconsin de Classificação de Cartas (TWCC), por grupo e por momento, comparações e significância.

\begin{tabular}{|c|c|c|c|c|c|c|c|}
\hline & & Pré & Pós & $\begin{array}{c}p \\
\text { Pré }\end{array}$ & Delta\% & $\begin{array}{l}\text { P } \\
\text { Pré- } \\
\text { pós }\end{array}$ & $\begin{array}{l}\text { Interação } \\
\text { grupo vs } \\
\text { momento }\end{array}$ \\
\hline \multirow{3}{*}{$\begin{array}{l}\text { Estado } \\
\text { Cognitivo } \\
\text { Global - } \\
\text { MoCa } \\
\text { (pontuação) }\end{array}$} & & & & & & & \\
\hline & $\mathrm{COM}$ & $23,1 \pm 4,7$ & $\begin{array}{l}25,1 \pm 3 \\
8\end{array}$ & 0,85 & $12,0 \pm 23,2$ & 0,003 & 0,98 \\
\hline & EB & $23,1 \pm 4,4$ & $\begin{array}{l}25,1 \pm 3 \\
9\end{array}$ & & $9,9 \pm 10,1$ & 0,001 & \\
\hline \multirow{2}{*}{$\begin{array}{l}\text { Abstração } \\
\text { TWCC } \\
\text { Categorias }\end{array}$} & $\mathrm{COM}$ & $2,3 \pm 0,8$ & $3,3 \pm 1,4$ & 0,08 & $58,7 \pm 87,1$ & \multirow{2}{*}{$\begin{array}{c}0,004 \\
0,24\end{array}$} & \multirow[t]{2}{*}{0,65} \\
\hline & EB & $2,5 \pm 1,5$ & $2,8 \pm 1,4$ & & $30,1 \pm 80,8$ & & \\
\hline \multirow{2}{*}{$\begin{array}{l}\text { Flexibilidade } \\
\text { Mental - } \\
\text { TWCC - } \\
\text { Erros } \\
\text { Perseverativos }\end{array}$} & $\mathrm{COM}$ & $12,8 \pm 8,7$ & $8,4 \pm 6,8$ & 0,09 & $-12,76 \pm 8,6$ & 0,034 & \multirow[t]{2}{*}{0,001} \\
\hline & EB & $\begin{array}{l}17,4 \pm 11 \\
3\end{array}$ & $\begin{array}{l}21,4 \pm 9 \\
8\end{array}$ & & $17,35 \pm 11,3$ & 0,11 & \\
\hline
\end{tabular}




\section{DISCUSSÃo}

O objetivo do presente estudo foi analisar e comparar possíveis efeitos, nas funções cognitivas de idosos, de dois protocolos de intervenção, um deles incluindo o SSE e o outro não.

$O$ primeiro resultado importante encontrado foi que o treinamento combinado de exercícios básicos e SSE parece ser mais efetivo para a cognição quando comparado com um treinamento exclusivamente com exercícios básicos. Assim, o SSE mostrou ser uma alternativa de exercício físico de baixo custo e com boa influência na cognição da população idosa.

Há que se ressaltar que ambos os grupos melhoraram a pontuação no MoCA, mostrando o efeito positivo da prática das atividades físicas no estado cognitivo global, independentemente do tipo de treinamento utilizado. O MoCA apresenta ênfase maior na avaliação de tarefas que requerem funcionamento executivo e de atenção (GUERREIRO, 2010) e há evidências de que a prática de exercícios físicos tem influência maior nas funções executivas de pessoas idosas, comparadas com a memória (COLCOMBE; KRAMER, 2003; SCHERDER et al., 2006).

Essa melhora no estado cognitivo global deve ser observada com cautela, pois pode ter ocorrido por melhora em funções específicas. Para avaliar funções específicas, utilizamos outro teste cognitivo, o TWCC. No presente estudo, enfocamos a abstração e a flexibilidade mental, constructos das funções executivas, avaliados pelo TWCC.

Observando os resultados no TWCC, o COM favoreceu melhoras nos subitens de funções executivas de abstração (categoria) e de flexibilidade mental (erros perseverativos), enquanto o EB melhorou em abstração e permaneceu inalterado em relação à flexibilidade mental. Tais achados mostram, novamente, benefícios da prática regular de atividade física, independente do tipo de treinamento. Contudo, a intervenção COM parece apresentar benefícios mais amplos. Esses resultados, originais quanto ao SSE, ganham maior relevância se atentarmos para o fato de que a perseveração do pensamento e a dificuldade de inibição de respostas inadequadas 
(características de redução da flexibilidade mental) causam aderência de determinada resposta à tarefa, o que leva a uma deficiência na resolução de problemas (KELLER; WERLANG, 2005).

A melhora dos grupos de intervenção em uma ou mais funções executivas corrobora os resultados de outros estudos que utilizaram como intervenção o exercício físico (COLCOMBE; KRAMER, 2003; Van GELDER et al., 2004; SCHERDER et al., 2005; COLCOMBE et al., 2004). Uma possibilidade de explicação poder ser a associação de um eventual ganho cardiovascular provocado pelo exercício à melhora das funções executivas. Conquanto não tenha sido escopo do presente estudo em analisar eventuais ganhos cardiovasculares, tal explicação é muito improvável em relação à intervenção com o SSE devido à forma de como foi desenvolvido (oito participantes por tapete), que não impunha uma significativa exigência cardiovascular, a ponto de aumentar a capacidade aeróbia de seus praticantes. Tal explicação pode ser mais plausível na intervenção com EB, por menores intervalos entre exercícios, e, portanto, mais frequência de esforços, comparada com a realização do SSE.

A melhora também da flexibilidade mental com o SSE, pode ter sido inerente à demanda maior de controlar respostas inadequadas ou corrigi-las para a execução correta da sequência mostrada pelo orientador, comparada com a execução de exercícios básicos. Em outras palavras, a melhora em funções executivas pode ser independente de eventual ganho cardiovascular proporcionado pelo exercício (SMILEY-OYEN et al., 2008). Assim, outros mediadores biológicos podem ser modulados pelo exercício e beneficiar a função neurocognitiva, incluindo vascularização cerebral, neurogênese e regulação dos fatores neurotróficos, independente do nível de condicionamento aeróbio (ETNIER et al., 2006; KRAMER; ERICKSON, 2007).

O SSE é apresentado como uma nova modalidade que utiliza estimulação motora (SHIGEMATSU; OKURA, 2006, SHIGEMATSU et al., 2008 a,b) aliada a uma demanda cognitiva significante, que o enriquece como possibilidade de utilização para diferentes populações. Nessa perspectiva, vários estudos (MATSUDO et al., 2001; MARTINSON et al., 2003; MAZO et al., 2004; PAPP et al., 2009; LUSTIG et al., 2009) enfatizam que devem ser exploradas intervenções que transcendam influência positiva apenas na função 
física ou mesmo cognitiva especificamente treinada, mas que tenham impacto também no estado cognitivo global (como mostrado no presente estudo) e na funcionalidade diária.

Finalizando, de forma original, o presente estudo mostra a efetividade da inserção do SSE combinado com exercícios básicos em funções cognitivas, abrindo possibilidades de desenvolvimento de estudos com tal intervenção em outros segmentos populacionais, particularmente em pessoas com declínio cognitivo leve ou que por outras razões temporárias ou infelizmente progressivas apresentem declínio cognitivo (por exemplo pessoas com doença de Alzheimer, no estágio inicial). Ainda, é importante investigar o efeito do SSE isoladamente, ou seja, sem combiná-lo com outros tipos de exercícios.

\section{REFERÊNCIAS}

AMERICAN COLLEGE OF SPORTS MEDICINE. American College of Sports Medicine position statement: Exercise and Physical Activity for Older Adults. Medicine \& Science in Sports \& Exercise, v. 41, n. 7, p. 1510-30, 2009.

ANTUNES, H.K.M. et al.Exercícios físico e função cognitiva: uma revisão. Revista Brasileira de Medicina do Esporte.v.12, n.2, p.108-114, 2006.

ANTUNES, H.K.M.; SANTOS, R.F.; HEREDIA, R.A.G.; BUENO; O.F.A., MELO, M.T. de. Alterações Cognitivas em idosas decorrentes do Exercício Físico Sistematizado. Revista da Sobama. v. 6, n.1, pp. 27-33, 2001.

BENEDETTI, T. R. B. et al..Atividade física e estado de saúde mental de idosos. Revista de Saúde Pública, São Paulo, v. 42, n. 2, p. 302-307, 2008.

BERG, E.A. A simpleobjectivetest for measuringflexibility in thinking. Journalof General Psychology, v. 39, p. 15-22, 1948. 
BRUCKI, S.M.D. Mini-Exame do Estado Mental: influência da escolaridade sobre os escores total e sub-itens. Revista de Neurociências. v. 4, p. 15-20, 1996.

BURGESS, P.W.et al. The ecological validity of tests of executive function. Journal of the International Neuropsychological Society, v. 4, p. 547-558, 1998.

BUTLER, R.N., FORETTE, F., GREENGROSS, B.S. Maintaining cognitive health in an ageing society.The Journal of The Royal Society for the Promotion of Health (JRSH), v.124, n.3, p;119-121, 2004.

CASSILHAS, R.C. et al. The Impact $f$ Resistance Exercise on the Cognitive Function of the Elderly. Medicine \& Science In Sport \& Exercise. v. 39, n. 8, p. 1401-07, 2007.

CHRISTOFOLETTI, G. et al. A controlled clinical trial on the effects of motor intervention on balance and cognition in institutionalized elderly patients with dementia. Clinical Rehabilitation, v. 22, p. 618-626, 2008.

COLCOMBE, S.; KRAMER, A.F.; Fitness effects on the cognitive function of older adults: a meta-analytic study. Psychological Science. v. 4, n. 2, 125-130, 2003.

COLCOMBE, SJ. et al. Cardiovascular fitness, cortical plasticity, and aging. PHYSIOLOGY.V.101, n.9, p. 3316-3321, 2004.

ETNIER, J.L. et al.A meta-regression to examine the relationship between aerobic fitness and cognitive performance.BrainResearchReviews. V. 52, p. 119-130, 2006.

GOBBI, S. Atividade física para pessoas idosas e recomendações da Organização Mundial de Saúde. Revista Brasileira de Atividade Física e Saúde, Londrina, v. 2, n. 2, p.41-49, 1997. 
GONÇALVEZ, R, GURJÃO, A.L.D, GOBBI, S. Efeitos de oito semanas do treinamento de força na flexibilidade de idosos. RevBras de Cineantropom Desempenho Hum. V. 9, n.2, p. 145-153, 2007.

GUERREIRO, M. Testes de rastreio de defeito cognitivo e demência: uma perspectiva prática Revista Portuguesa de Clinica Geral. v. 26, p. 46-53, 2010.

GUIMARÃES, L. H. C. T. et al. Avaliação da capacidade funcional de idosos em tratamento fisioterapêutico. Revista Neurociências, São Paulo, v. 12, n. 3 , p. 130-133, 2004.

KELLER, M.; WERLANG, B. S. G. Flexibilidade na resolução de problemas em tentadores de suicídio. Jornal Brasileiro de Psiquiatria, v. 54, n. 2, p. 128136, 2005.

KRAMER, AF; ERICKSON, KI. Capitalizing on cortical plasticity: influence of physical activity on cognition and brain function. Cognitive Science, v.11, n. 8 , p, 342-348, 2007.

LAURIN, D. et al. Physical activity and risk of cognitive impairment and dementia in elderly persons. Archives of Neurology. v. 58, p. 498-504, 2001.

LEZAK, M.D.; HOWLESON, D.B.; LORING, D.W. Neuropsychological Assessment.4. Ed. New York, NY: Oxford University Press, 2004.

LUSTIG, C. et al. Aging, training, and the brain: a review and futures directions. Neuropsychology review.19, p. 504-522, 2009.

MARTINSON, B. et al. Changes in physical activity and short-term changes in health care changes: a prospective cohort study of older adults. Preventive Medicine, San Diego, v. 37, p. 319-326, 2003. 
MATSUDO, S. M.; MATSUDO, V. K. R.; NETO, T. L. B. Atividade física e envelhecimento: aspectos epidemiológicos. Revista Brasileira de Medicina do Esporte, São Paulo, v. 7, n. 1, p. 2-13, 2001.

MAZO, G.Z. et al. Aptidão funcional geral e índice de massa corporal de idosas praticantes de atividade física. Rev. Bras.Cineantropom. Desempenho Hum. V. 8, n. 4, p.46-51, 2006.

NELSON, H.A modified card sorting test sensitive to frontal lobes defects.Cortex, v. 12, p. 313-324, 1976.

PAPP, KV, WALSH, SJ, SNYDER, PJ. Immediate and delayed effects of cognitive interventions in healthy elderly: $A$ review of current literature and future directions. Alzheimer's \& Dementia: The Journal of the Alzheimer's Association, v.5, n.1, p 50-60, 2009.

SCHERDER, EJ. et al. Physical activity and executive functions in the elderly with mild cognitive impairment. Aging and Mental Health v.9, p. 272-280, 2005.

SEBASTIÃO, E. et al. Efeitos da prática regular de dança na capacidade funcional de Mulheres acima de 50 anos. Revista da Educação Física/UEM Maringá, v.19, n. 2, p. 205-214, 2008.

SHIGEMATSU, R.; OKURA, T. A novel exercise for improvinglowerextremityfunctional fitness in theelderly. Aging Clinical and Experimental Research.v. 18, n 3, p. 242-248, 2006.

SHIGEMATSU, R. et al. Square-Steping Exercise and Fall Risk Factors in Older Adults: A Single-Blind, Randomized Controlled Trial. Journal of Gerontology: Medical Sciences, v. 63A, n 1, p.76-82, 2008a. 
SHIGEMATSU, R. et al. Square Stepping Exercise versus strength and balance training for fall risk factors. Aging Clinical and Experimental Research.v. 20, $\mathrm{n}$ 1, p. 19-24, 2008b.

SMILEY-OYEN, AL. et al. Exercise, fitness, and neurocognitive function in older adults: the selective inprovement" and "cardiovascular fitness" hypotheses. Ann. Behav. Med. V.36, p.280-291, 2008.

SMITH, T.; GILDEH, N.; HOLMES, C. The Montreal Cognitive Assessment: Validity an Utility in a Memory Clinic Setting. The Canadian Journal of Psychiatry, v. 52, n 5, 2007.

SPREEN, O.; STRAUSS, E.A compendium of neuropsychological tests. New York: Oxford University Press, 1991.

VANCE, D. et al.The effects of physical activity and sedentary behaviour on cognitive health in older people.Journal of Aging Physical Activity.v.13, p. 294-313, 2005.

Van Gelder BM. et al. Physical activity in relation to cognitive decline in elderly men: The FINE study. Neurology, v. 63, p. 2316-21, 2004;

VERGHESE, J. et al. Leisure activities and the risk of dementia in the elderly.The New England Journal of Medicine.v.348, p. 2508-16, 2003.

VOORRIPS, L. et al.A physical activity questionnaire for elderly.Medicine and Science in Sports and Exercise, v. 23, n. 8, p. 974-979, 1991. 


\section{SQUARE STEPPING EXERCISE AND BASIC EXERCISES ON COGNITION IN ELDERY}

\section{ABSTRACT}

The aim of thisstudy was tocompare the cognitive functionof older people pre and post twointervention protocols: basic exercisescombined to theSquareSteppingExercise (COM) andbasic exercises alone(EB). All participants were evaluatedbefore and after4 months ofintervention by means of MontrealCognitive Assessment(MoCA) and WisconsinSortingCard Test (TWCC). The results showed improvement in both groupsregarding the global cognitive status, but onlyCOMshowed specificimprovementson abstractionand mental flexibility. Thus, combined trainingof basic exercisesand SSEseems to bemore effectivefor cognitionwhen comparedwith trainingexclusively based on basic exercises.

Key-Words: Aged people. Cognition.Physicalexercise.

\section{SQUARE STEPPING EXERCISE Y EJERCICIOS BASICOS SOBRE LA COGNICI[ON EN ADULTOS MAYORES}

\section{RESUMEN}

El objetivo disteestudio fue compararla función cognitiva de ancianosantes y después dosprotocolos de intervención: ejercicios básicosrelacionados conel SquareSteppingExercise(COM) y ejerciciossólo de base(EB). Todos fueron evaluadosantes y después de4meses de intervencióna través delMontréalCognitiveAssessiment(MOCA) y eltest de clasificación decartas de Wisconsin(TWCC). Los resultados mostraron queambos gruposmuestran una mejoría enel estado cognitivoglobal, pero queCOM sólomostraron una mejoría específicaenla abstracción y flexibilidad mental. Así,el entrenamiento combinadodeejercicios básicosySSEparece ser máseficazpara la cognicióncuando se comparaconunaformaciónexclusivamenteconejercicios básicos.

Palabras clave: Ancianos. Cognición. Ejercicio físico. 